\title{
Photonic crystals for optimal color conversion in light-emitting diodes: a semi-analytical approach
}

\author{
Constantinos Valagiannopoulos ${ }^{1, *}$ (1) and Pavlos G. Lagoudakis ${ }^{2,3}$ \\ ${ }^{1}$ Department of Physics, School of Science and Technology, Nazarbayev University, 53 Qabanbay Batyr Ave, Astana KZ-010000, Kazakhstan \\ ${ }^{2}$ Department of Physics and Astronomy, University of Southampton, Southampton SO17 1BJ, UK \\ ${ }^{3}$ Skolkovo Institute of Science and Technology, 100 Novaya St, Skolkovo 143025, Russian Federation \\ *Corresponding author: konstantinos.valagiannopoulos@nu.edu.kz
}

Received 5 February 2018; revised 10 March 2018; accepted 14 March 2018; posted 19 March 2018 (Doc. ID 321427 ); published 13 April 2018

\begin{abstract}
Based on effective-medium approximation, we analytically optimize the color conversion of GaN-based lightemitting p-n junctions, with respect to the size and spatial density of the used photonic crystals (PhCs), being filled with quantum dots (QDs). The model is two-dimensional and the incoherent excitation into the multiple quantum wells is mimicked by line sources of random electric and magnetic currents. Optimal operation is achieved by suppressing the radiation extraction for one visible color and simultaneously maximizing the emission for another color. For these cases, the field gets inevitably concentrated into the PhCs of QDs, where the conversion occurs, and the performance of the device is maximized. Several examples from these ultra-performing designs are provided, within which a secondary sweep can be applied by posing extra constraints related to cost or ease of fabrication. () 2018 Optical Society of America
\end{abstract}

OCIS codes: (040.4200) Multiple quantum well; (050.2065) Effective medium theory; (230.3670) Light-emitting diodes; (230.7405) Wavelength conversion devices; (350.4238) Nanophotonics and photonic crystals.

https://doi.org/10.1364/JOSAB.35.001105

\section{INTRODUCTION}

Light-emitting diodes (LEDs) have a long history starting from the early 1960s, when scientists working on semiconductors realized that the forward current through a common $\mathrm{p}-\mathrm{n}$ diode results in electron-hole recombination and inevitably in release of photons due to the difference in energy levels [1,2]. This old design has recently undergone numerous improvements, making its use as ubiquitous as the screen incorporated in numerous devices [3]. In particular, the increased necessity for colorful high-quality projection dictated the transition from LEDs producing white or monochromatic light [4] to setups with color-selective emission across the entire visible spectrum. Such color-sensitive operation is successfully supported by quantum dots (QDs), namely, small semiconducting particles with highly tunable emitting frequency as long as their size affects substantially the neighboring effective optical properties (quantum confinement).

The operating mechanisms of components containing such QDs and the key advantages of using them as luminophores in LEDs have been thoroughly described [5], with emphasis on their size-controllable response owing to their colloidal nature. Quantum-dot photophysics has been also used [6] to build structures with high electroluminescence efficiencies and peak luminance values exploiting strong electronic coupling. Furthermore, alternative designs of QD-based LEDs with solution-processed multilayers have been reported [7] to exhibit color-saturated deep-red emission and a number of additional advantages such as high external quantum efficiencies, lowefficiency roll-off, and long operational lifetime. Other beneficial characteristics (high quantum yields, narrow emission, low exciton binding energies) are acquired to color tunable light emitters incorporating QDs with use of perovskites [8]. Nonetheless, the aforementioned designs possess a major weakness: the fascinating coloring processes described concern only the small portion of the power that escapes the device; on the contrary, most of the emitted energy remains in the diode due to total internal reflection. To remedy such a defect, one can modify the structure with additional patterned layers where nanocones [9] or spherical particles [10], made of oxides, are deposited.

However, the most common way to enhance the light extraction from a planar LED configuration is the use of periodic clusters of parallel cylinders: the so-called photonic crystals (PhCs). Such a structural change alters the frequency-dispersive effective behavior of the background medium by creating bandpass and band-stop zones; therefore, $\mathrm{PhC}$ have become an important class of materials with great potential for applications ranging from medicine to electronic and optoelectronic devices $[11,12]$. An experimental demonstration of the ability of $\mathrm{PhCs}$ to inhibit the spontaneous emission of light and enable 
directionally and polarization-independent photonic bandgaps has been announced [13], while the same principle has been utilized to enhance the external radiation [14].

The extracted power after color conversion in LED structures gets additionally amplified with use of photonic crystals; in particular, the most common technique is to fill these periodically positioned cylinders (PhCs) with quantum dots. Enhanced light emission in free space has been reported in structures incorporating QD-loaded photonic crystals through the proper phase matching of the developed leaky modes $[15,16]$. Furthermore, Fabry-Perot and PhC-induced modes are observed in the far-field radiation patterns [17] leading to substantial light generation, while increased coloring efficiency utilizing non-radiative energy transfer is demonstrated in LED configurations [18]. Epitaxial structures are also extensively used to modify the distribution of guided modes [19] or boost internal quantum efficiency [20]. Finally, hybrid designs with nanopillars of QDs embedded into multiple quantum wells (QWs) are found to achieve record-high performance in wireless power transfer [21] and color conversion [22].

In this work, we consider an LED configuration similar to the ones just described, namely, a planar diode with p- and n-doped GaN layers sandwiching multiple quantum wells loaded with photonic crystals and filled by quantum dots. We employ quasi-static approximation [23] for the effective constituent parameters of the layers containing QWs and $\mathrm{PhCs}$. Accordingly, our problem becomes analytically [24] solvable and we obtain rigorous expressions for the radiated light intensity under the incoherent excitation [25] supplied by the QWs. These formulas can give us a reliable indicator of the device's light-extraction efficiency as a function of the operating frequency, with very low computational cost. In this way, we can run fast optimizations to detect configurations which suppress the emission for one color and enhance the radiation for another color. In particular, a structure of photonic crystals which makes LED perform poorly at the blue-color frequency and favors red-color extraction, will exhibit an excellent blue-to-red color conversion as long as the features of the QD particles permit. Indeed, blue photons are absorbed by the QDs and re-emitted in the form of red photons; therefore, the proposed optimal configurations will "force" the field to concentrate into the QDs, where the trapped blue light has the unique chance to transform into red. The conditions for the period of the $\mathrm{PhC}$ lattice and the radius of its cylinders are numerically shown so that maximal color conversion occurs.

\section{SEMI-ANALYTICAL MODEL}

\section{A. Structure, Texture, and Objective}

Two simple sketches of the light-emitting structure under investigation are shown in Fig. 1, where the employed Cartesian coordinate system $(x, y, z)$ is also defined. In Fig. 1(a), we show multiple $(N)$ stacked bilayers of $\mathrm{GaN}$ (relative permittivity $\varepsilon_{g}$ and thickness $d_{g}$ ) and InGaN (relative permittivity $\varepsilon_{i}$ and thickness $d_{i}$ ), which form the multiple quantum wells, every single point of which can act as a dipole source to produce the incoherent radiation which feeds the device [7]. A detailed view of a single bilayer of the QWs is depicted in the lower right inset of Fig. 1(a). On the top of these multilayers, a slab of

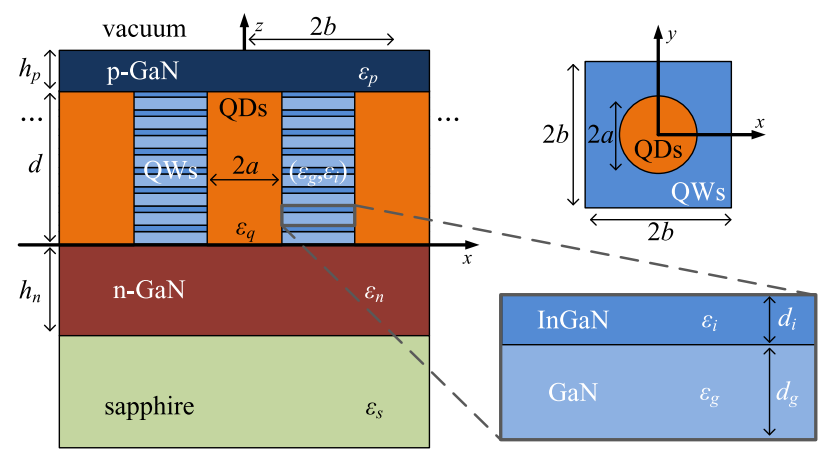

(a)

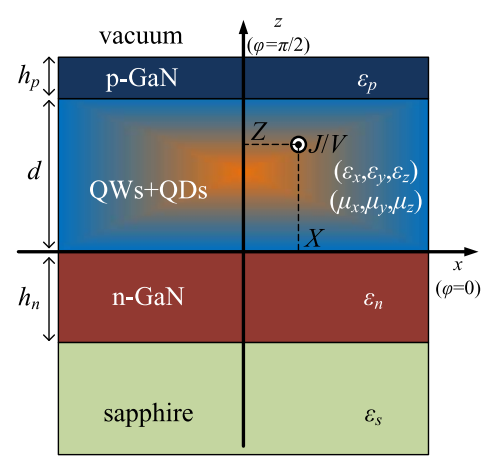

(b)

Fig. 1. (a) A simplistic sketch of the LED physical configuration. Multiple bilayers of $\mathrm{GaN}$ and $\mathrm{InGaN}$ (shown in detail at the bottom inset) constitute the quantum wells (QWs), which are loaded with photonic crystals (PhCs) of circular cylindrical cross section filled with quantum dots (QDs). The structure is grown on a sapphire base covered by a thick layer of $\mathrm{n}$-doped $\mathrm{GaN}$. In the upper inset, we show the dimensions of a single square cell of the photonic crystal lattice. (b) Same sketch as in Fig. 1(a), but now the multilayers of QWs with the QDs cylinders have been replaced by a single slab of the same size and homogeneous texture.

p-doped GaN (permittivity $\varepsilon_{p}$ ) is grown to have height $h_{p}$. The QWs are loaded by a square lattice of photonic crystals filled with quantum dots whose cross section is a circle of radius $a$, while its spatial period equals $2 b$ (with $a<b$ ); obviously, the color conversion occurs in these QDs [1]. The top view of a single cell (square with dimensions $2 b \times 2 b$ ) is shown in the upper right inset of Fig. 1(a). The structure of QWs with the QD cylinders has been grown on a sapphire half-space (relative permittivity $\varepsilon_{s}$ ) covered by a layer of n-doped GaN (relative permittivity $\varepsilon_{n}$ ) with thickness $h_{n}$. In this way, we have defined a planar $\mathrm{p}-\mathrm{n}$ diode of $\mathrm{GaN}$ which is excited by the random dipoles of QWs and performs the LED operation into the cylinders of QDs. The configuration can have extra layers $\left(\mathrm{TiO}_{2}\right.$ or alternative quasi-transparent oxides [6]) which the cylinders may perforate for practical fabrication purposes; such modifications will only trivially increase the complexity of the device, and thus we opted to consider the simplest possible design. Similar $\mathrm{p}-\mathrm{n}$ structures assisted by photonic crystals have been reported as functional and experimentally verified $[15,21,22]$.

In Fig. 1(b), we show the two-dimensional (2D) and analytically solvable equivalent of the configuration of Fig. 1(a); 
here, the multilayers of QWs loaded with QD photonic crystals is substituted by a homogeneous slab of the same thickness $d=N\left(d_{g}+d_{i}\right)$ characterized by diagonal tensors of relative permittivity $[\varepsilon]=\operatorname{diag}\left(\varepsilon_{x}, \varepsilon_{y}, \varepsilon_{z}\right)$ and permeability $[\mu]=$ $\operatorname{diag}\left(\mu_{x}, \mu_{y}, \mu_{z}\right)$. The incoherent excitation can be emulated by summing [25] the developed field intensities from $2 \mathrm{D}$ electric and magnetic dipoles positioned along an arbitrary axis $(x, z)=(X, Z)$ parallel to the $y$ axis, with electric $J$ (Ampere) or magnetic $V($ Volt $)$ currents respectively having random magnitude and phase. These emitting sources can be placed everywhere into the slab $0<z<d$, excluding the regions occupied by the QDs.

The energy, pumped by the incoherently radiating dipoles, will be provided to the QDs and LED action will take place. In particular, the current flowing through the photonic crystal will lead to absorption of higher-frequency (blue) light and reemission of lower-frequency (red) light. The $\mathrm{p}-\mathrm{n}$ junction is created between the $\mathrm{n}-\mathrm{GaN}$ layer and the $\mathrm{p}-\mathrm{GaN}$ slab, which is deposited on the QWs $(z>d)$ shown in Fig. 1. In this way, we have the electron-hole recombination for blue light into the QD-filled cylinders, and accordingly the release of photons will create the red light into vacuum. Our objective is to select configurations which favor this color conversion and the radiation extraction into free space $z>d+h_{p}$.

The time dependence is considered harmonic of frequency $\omega: \exp (+j \omega t)=\exp \left(+j k_{0} c t\right)$ and suppressed throughout the analysis. The symbol $k_{0}=2 \pi / \lambda_{0}$ is used for the wavenumber, $\lambda_{0}$ is the operating wavelength, $\eta_{0}$ is the wave impedance, and $c$ is the speed of light, all into vacuum.

\section{B. Effective Permittivities and Permeabilities}

In order to determine the elements of tensors $[\varepsilon]$ and $[\mu]$, we first consider the multiple QWs in the absence of the photonic crystal cylinders $(a=0)$ and we replace them, due to the very small size of their bilayers $\left(\max \left\{d_{g}, d_{i}\right\} \ll \lambda_{0} /\right.$ $\left.\sqrt{\max \left\{\left|\varepsilon_{g}\right|,\left|\varepsilon_{i}\right|\right\}}\right)$, with a homogeneous background medium having one transversal ( $t$, across $x y$ plane) and one longitudinal ( $l$, along $z$ axis) relative permittivity given by [26]

$$
\varepsilon_{t}=\frac{d_{g} \varepsilon_{g}+d_{i} \varepsilon_{i}}{d_{g}+d_{i}}, \quad \varepsilon_{l}=\frac{\left(d_{g}+d_{i}\right) \varepsilon_{g} \varepsilon_{i}}{d_{g} \varepsilon_{i}+d_{i} \varepsilon_{g}} .
$$

The magnetic permeability of the multiple QWs is kept equal to unity since the participating media are magnetically inert. It should be stressed that the permittivities $\varepsilon_{g}, \varepsilon_{i}$ do not differ substantially from each other and thus the same happens with the corresponding effective $\varepsilon_{t}, \varepsilon_{l}$ ones.

To this end, the complex structure occupying the region $0<z<d$ can be further homogenized, namely, substituted by a single slab of identical thickness $d$ whose electromagnetic interactions are determined simply by two diagonal tensors of effective permittivities and permeabilities. By employing the so-called quasi-static approximation [23], these parameters are given by

$$
\begin{aligned}
& \varepsilon_{x}=\varepsilon_{y}=\varepsilon_{t}\left[1+2 f \frac{\left(\varepsilon_{q} / \varepsilon_{t}\right) g\left(k_{0} a \sqrt{\varepsilon_{q}}\right)-1}{\left(\varepsilon_{q} / \varepsilon_{t}\right) g\left(k_{0} a \sqrt{\varepsilon_{q}}\right)+1}\right], \\
& \varepsilon_{z}=\varepsilon_{l}\left[1+f \frac{\left(2 \varepsilon_{q} / \varepsilon_{l}-1\right) g\left(k_{0} a \sqrt{\varepsilon_{q}}\right)-1}{g\left(k_{0} a \sqrt{\varepsilon_{q}}\right)+1}\right]
\end{aligned}
$$

$$
\begin{aligned}
& \mu_{x}=\mu_{y}=\frac{1+f\left[g\left(k_{0} a \sqrt{\varepsilon_{q}}\right)-1\right] /\left[g\left(k_{0} a \sqrt{\varepsilon_{q}}\right)+1\right]}{1-f\left[g\left(k_{0} a \sqrt{\varepsilon_{q}}\right)-1\right] /\left[g\left(k_{0} a \sqrt{\varepsilon_{q}}\right)+1\right]}, \\
& \mu_{z}=1+f \frac{g\left(k_{0} a \sqrt{\varepsilon_{q}}\right)-1}{g\left(k_{0} a \sqrt{\varepsilon_{q}}\right)+1} .
\end{aligned}
$$

The notation $f=\frac{\pi a^{2}}{4 b^{2}}$ is used for the filling factor of the QDs into the square cell of the QWs; it is directly proportional to the (square of) the ratio $a / b$. The auxiliary function $g(x)$ is defined as $g(x)=\frac{J_{1}(x)}{x J_{1^{\prime}}(x)}=\frac{J_{1}(x)}{x J_{0}(x)-J_{1}(x)}$, where $J_{n}$ is the $n$-th order Bessel function.

The approximation indicated by Eqs. (2) and (3) is far more robust and sophisticated that the static one of Eq. (1), and that is why we are using it under less strict homogenization constraints. In other words, we will employ Eqs. (2) and (3) even when the size of the cylinders is not much smaller than the wavelength into the background medium of QWs [defined through Eq. (1)] as dictated by standard effective-medium averaging principles $[27,28]$. The presence of Bessel functions in the definition of $g(x)$ indicates that the resonances of the cylindrical particles are captured by their own variation; therefore, we believe that the approximation can give meaningful results even if the homogenization demand $\left.2 a \ll \lambda_{0} / \sqrt{\max \left\{\left|\varepsilon_{t}\right|,\left|\varepsilon_{l}\right|\right.}\right\}$ is not valid. Further evidence that the utilized quasi-static approximation is fairly wise is that it predicts magnetic response [29] for the equivalent structure $\left(\mu_{x}, \mu_{y}, \mu_{z} \neq 1\right)$ due to the spatial variation of electric current even though the relative permeabilities of the used materials are all equal to 1 . To put it alternatively, the geometry of the photonic crystals and the way that the fields vary at the inner and outer regions of each circular boundary are taken into account when evaluating the effective permeabilities of Eq. (3). Of course, the aforementioned technique is an approximate one but we advocate that, due to the fact that it renders the problem analytically solvable, it can give useful insight into the underlying physics and lighten substantially the computational burden for the design optimizations.

More specifically, with respect to the tradeoff between simplicity of analysis and applicability, the proposed model takes the first option. However, the obtained results are still able to capture the effects of the main factors of the mechanisms that govern the physical phenomena that occur. To put it alternatively, we aim at providing good initial parametric guesses for optimal designs and not exact values for all the structural sizes; a local scanning around these initial points for the maximum performance can be done by computationally heavy but more accurate techniques [30,31]. Note also that the formulas (2) and (3) are derived for an isotropic background (namely, for the case $\varepsilon_{t}=\varepsilon_{l}$ ) but are still physically sound in our consideration because $\varepsilon_{t} \cong \varepsilon_{l}$, as mentioned previously. Furthermore, for the transversal permeabilities $\mu_{x}, \mu_{y}$ we use a finer approximation [32] than the one derived in the work from which Eqs. (2) and (3) are reproduced [23]. It takes into account the developed Floquet-Bloch waves into the photonic crystal and diversifies axial direction $z$ not only for the electric $\left(\varepsilon_{z} \neq \varepsilon_{x}, \varepsilon_{y}\right)$ but also for the magnetic $\left(\mu_{z} \neq \mu_{x}, \mu_{y}\right)$ response. 


\section{Analytical Solution}

The primary fields of the two-dimensional dipoles [shown in Fig. 1(b)], either electric (line current $J$ measured in Ampere) or magnetic (line current $V$ measured in Volt), can be expressed into an effective medium with diagonal permittivity and permeability tensors $[\varepsilon]=\operatorname{diag}\left(\varepsilon_{x}, \varepsilon_{y}, \varepsilon_{z}\right)$ and $[\mu]=$ $\operatorname{diag}\left(\mu_{x}, \mu_{y}, \mu_{z}\right)$ in the form of spectral integrals [33]. More specifically, the electric dipole produces a TE field distribution with a sole $y$ electric component, and the magnetic dipole develops a TM field with magnetic field parallel to the $y$ axis. The corresponding formula in mutual notation is given by

$$
\mathbf{F}_{\mathrm{TE} / \mathrm{TM}}^{\mathrm{prim}}=\hat{\mathbf{y}} \frac{A_{\mathrm{TE} / \mathrm{TM}}}{4 \pi j} \int_{-\infty}^{+\infty} \frac{e^{-|z-Z| \kappa_{\mathrm{TE} / \mathrm{TM}}(\beta)}}{\kappa_{\mathrm{TE} / \mathrm{TM}}(\beta)} e^{j \beta(x-X)} \mathrm{d} \beta,
$$

where $\mathbf{F}^{\text {prim }}$ denotes the background electric field of a dipole positioned along the axis $(x, z)=(X, Z)$ for the TE polarization and the respective magnetic field for the TM one. Furthermore, $A_{\mathrm{TE}}=k_{0} J \mu_{x} \eta_{0}$ and $\kappa_{\mathrm{TE}}=\sqrt{\mu_{x}\left(\beta^{2}-k_{0}^{2} \varepsilon_{y} \mu_{z}\right)} /$ $\sqrt{\mu_{z}}$, while $A_{\mathrm{TM}}=k_{0} V \varepsilon_{x} / \eta_{0}$ and $\kappa_{\mathrm{TM}}=\sqrt{\varepsilon_{x}\left(\beta^{2}-k_{0}^{2} \varepsilon_{z} \mu_{y}\right)} /$ $\sqrt{\varepsilon_{z}}$. The decoupling of waves into TE and TM types is a fundamental feature of Maxwell's equations and has been adopted in a similar manner [34] when considering LED structures.

After imposing the necessary boundary conditions [24] at $z=-h_{n}, 0, d,\left(d+h_{p}\right)$, the (radiating) electromagnetic field into free space $\left(z>d+h_{p}\right)$ can be determined as another spectral integral with the same notation for $\mathbf{F}$ as in Eq. (4): $\mathbf{F}_{\mathrm{TE} / \mathrm{TM}}^{\mathrm{rad}}=\hat{\mathbf{y}} \int_{-\infty}^{+\infty} S_{\mathrm{TE} / \mathrm{TM}}(\beta, Z) e^{-\sqrt{\beta^{2}-k_{0}^{2}} z+j \beta(x-X)} \mathrm{d} \beta$. The spectral profile functions $S_{\mathrm{TE} / \mathrm{TM}}(\beta, Z)$ are measured in different units for the two polarizations and are dependent only on the vertical $(Z)$ position of the source since the $X$-dependence $e^{j \beta(x-X)}$ is trivial. The exact expressions for the quantities $S_{\mathrm{TE} / \mathrm{TM}}(\beta, Z)$, which are proportional to the random electric/magnetic currents $J / V$ respectively, are omitted for brevity. By implementing the stationary phase method, the radiated field far from the structure (distance $r=\sqrt{x^{2}+z^{2}} \rightarrow \infty$ ) along the direction forming angle $\varphi$ with the positive $x$ semi-axis is written as [35]

$$
\begin{aligned}
\mathbf{F}_{\mathrm{TE} / \mathrm{TM}}^{\mathrm{rad}}(\varphi, X, Z) \cong & \hat{\mathbf{y}} S_{\mathrm{TE} / \mathrm{TM}}\left(-k_{0} \cos \varphi, Z\right) e^{j k_{0} X \cos \varphi} k_{0} \\
& \times \sin \varphi \sqrt{\frac{2 \pi j}{k_{0} r} e^{-j k_{0} r} .}
\end{aligned}
$$

Obviously, such a field propagates radially along each direction of the upper half-space $0<\varphi<\pi$, and the surface density of the electromagnetic power along each angle $\varphi$ can be computed with use of the Poynting theorem [36]:

$p_{\mathrm{TE} / \mathrm{TM}}(\varphi, Z)=C_{\mathrm{TE} / \mathrm{TM}} \frac{k_{0} \pi}{r}\left|S_{\mathrm{TE} / \mathrm{TM}}\left(-k_{0} \cos \varphi, Z\right)\right|^{2} \sin ^{2} \varphi$,

where $C_{\mathrm{TE}}=1 / \eta_{0}$ and $C_{\mathrm{TM}}=\eta_{0}$. It should be remarked that the power is not dependent on the horizontal position $X$ of the source, which can be anticipated due to the infinite dimensions of the structure across the $x y$ plane. Therefore, to find the average radiated power $P_{\mathrm{TE} / \mathrm{TM}}(\varphi)$ along angle $\varphi$ from all the sources positioned along the vertical strip $0<z<d$, it suffices to average [37] $p_{\mathrm{TE} / \mathrm{TM}}(\varphi, Z)$ over $0<Z<d$ with electric $J(Z)$ and magnetic $V(Z)$ excitation currents which are random complex (amplitude and phase) functions of the position $Z$. Of course, the incoherent dipole sources can exist only in the quantum well multilayers and not in the QD cylinders; therefore, we should multiply the integral of power densities by $(1-f)$, where $f$ is the filling factor of QDs into the effective layer of thickness $d$ :

$$
P_{\mathrm{TE} / \mathrm{TM}}(\varphi)=\frac{1-f}{d} \int_{0}^{d} p_{\mathrm{TE} / \mathrm{TM}}(\varphi, Z) \mathrm{d} Z .
$$

As far as the intensity $I_{\mathrm{TE} / \mathrm{TM}}$ of the radiated field for all the possible directions $0<\varphi<\pi$ is concerned, we can further aggregate [25] $P_{\mathrm{TE} / \mathrm{TM}}$ over the upper-half semi-circle of large radius $r \rightarrow+\infty$. Note that the total intensity is obtained as a sum (integral) of the (randomly initialized) intensities for every single source since the excitation is incoherent and the fields are not radiating simultaneously [37]. In particular,

$$
I_{\mathrm{TE} / \mathrm{TM}}=r \int_{0}^{\pi} P_{\mathrm{TE} / \mathrm{TM}}(\varphi) \mathrm{d} \varphi \text {. }
$$

The quantity $P_{\mathrm{TE} / \mathrm{TM}}(\varphi)$ is measured in Watt/meter ${ }^{2}$ and expresses the surface density of power sent to the far region on average by all the incoherently radiating dipoles (for the corresponding polarization type TE or TM) along the direction-making angle $\varphi$ with positive $x$ semi-axis on the $x z$ plane. The quantity $I_{\mathrm{TE} / \mathrm{TM}}$ is measured in Watt/meter and expresses the total far-field power produced by the random dipoles into QWs per unit length of $y$ axis (along all directions $\varphi)$. It is a significant advantage that the formulas (7) and (8) require only numerical integrations (with respect to $Z$ and $\varphi$ ) instead of simulating random radiation from every single point of the QWs via commercial software [30]. On the other hand, it should be stressed that only a portion of the electrons are recombined with holes into QDs in order to emit a photon. The rest of them are either trapped in a potential well, converted to vibrational energy of lattice atoms (phonons), or recombined at higher energy levels without radiation [3]. In the present study, we assume that the aforementioned radiative recombination rate [1] (and the corresponding quantum yield) remains constant before and after the placement of PhCs, this being an identity property of QWs where the recombination happens. Since we will depict variations of normalized quantities (radiation with PhCs over radiation without), these losses will not affect substantially our results.

\section{NUMERICAL RESULTS}

\section{A. Parameter Ranges and Effective Media}

The values for our model's parameters are obtained from the published work [22] that experimentally validates the concept of a $\mathrm{p}-\mathrm{n}$ diode loaded with PhCs. It is needless to say that alternative sizes and materials can be chosen, and the corresponding system will be treated identically; in this study, our intention is to demonstrate how the lattice of PhCs is optimized for an already tested background configuration (QWs, GaN, sapphire layers). In particular, the thickness of the n-doped $\mathrm{GaN}$ is huge $-h_{n} \cong 4500 \mathrm{~nm}$, contrary to the size of the $\mathrm{p}$-doped layer 
of the junction: $h_{p} \cong 220 \mathrm{~nm}$. As far as the multiple QWs are concerned, the heights of the layers are taken equal to a few nanometers, namely, $d_{g} \cong 12 \mathrm{~nm}$ and $d_{i} \cong 3 \mathrm{~nm}$, while the number of bilayers is kept moderate [22]: $N=8$; therefore, $d=N\left(d_{g}+d_{i}\right) \cong 120 \mathrm{~nm}$. It should be remarked that the response of the structure seems not strongly dependent on the vertical size $d$ of the slab with QD-filled PhCs (which is deeply subwavelength for wavelengths $\lambda_{0}$ belonging in the visible spectrum). Such a feature may be attributed to the nature of the sources; they incoherently radiate, being positioned along the whole $0<z<d$ line, and the total emission is an aggregation of the solutions' intensities for every single boundary value problem. Since we are interested in the color conversion into the described $\mathrm{p}-\mathrm{n}$ diode operating as LED, we mainly examine two operational wavelengths: one corresponding to blue light $\left(\lambda_{0}=450 \mathrm{~nm}\right)$ and another to red light $\left(\lambda_{0}=630 \mathrm{~nm}\right)$. For the former case (blue), the permittivities are given by $\varepsilon_{s} \cong 1.78^{2}, \varepsilon_{n} \cong 2.46^{2}, \varepsilon_{g} \cong 2.48^{2}, \varepsilon_{i} \cong 2.2^{2}$, $\varepsilon_{p} \cong 2.46^{2}$, and $\varepsilon_{q} \cong 1.69^{2}$. For the latter case (red), the permittivities are given by $\varepsilon_{s} \cong 1.77^{2}, \varepsilon_{n} \cong 2.36^{2}, \varepsilon_{g} \cong 2.38^{2}$, $\varepsilon_{i} \cong(2.32-j 0.1)^{2}, \quad \varepsilon_{p} \cong 2.36^{2}$, and $\varepsilon_{q} \cong(1.72-j 0.02)^{2}$. The material data is taken from well-established databases [38] and reports [39]. It can be noticed that the selection of two colors (blue, red) is related to the specific type of quantum dots and the sort of color conversion they support [22]. Exactly the same procedure as described subsequently could be followed for different choices of visible frequencies in which the corresponding coloring through QDs occurs.

In Fig. 2, we show the variation of the real parts of effective permittivities [Eq. (2)] and permeabilities [Eq. (3)] as functions of the ratio $a / b$ for blue light [Fig. 2(a), $\lambda_{0}=450 \mathrm{~nm}$ ] and red light [Fig. 2(b), $\lambda_{0}=630 \mathrm{~nm}$ ] excitation. The size of the cell is kept fixed: $b=110 \mathrm{~nm}$. Due to the small losses, we omit the variations of the imaginary parts. It is clear that in the smaller wavelength case (blue light), the effective constituent quantities (either permittivities or permeabilities, not both, for a specific pair of normal directions [40]) are taking negative values and therefore create bandgaps. Into them, the propagation is blocked or, more accurately, it happens to a negligible extent only due to the existence of small losses. The resonances occur at a similar $a / b$ for both the electric and magnetic transversal parameters and the same happens for the longitudinal ones. Under red color illumination [Fig. 2(b)], the variation is milder

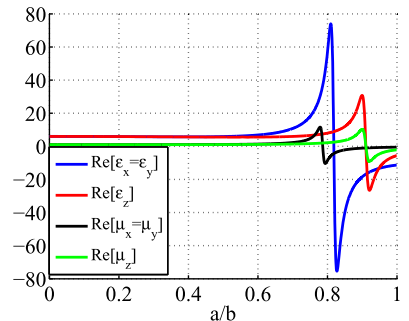

(a)

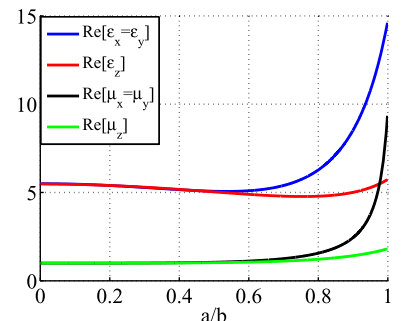

(b)
Fig. 2. Real parts of the effective permittivities and permeabilities of the homogenized layer comprised of multiple QWs and photonic crystals filled with QDs as functions of dimensions ratio $a / b$ for (a) $\lambda_{0}=450 \mathrm{~nm}$ (blue light) and (b) $\lambda_{0}=630 \mathrm{~nm}$ (red light). Plot parameters: $b=110 \mathrm{~nm}$. since the size of the cylinders is electrically smaller; one could also notice the almost isotropic nature of quantum wells when the photonic crystals are thin $(a<0.5 b)$, which is not easily discerned in Fig. 2(a).

In Fig. 3, we represent the intensities $I_{\mathrm{TE} / \mathrm{TM}}$ of the radiated field over the entire upper half space $0<\varphi<\pi$ (namely, for $z>d+h_{p}$ for both polarizations TE and TM), which are normalized by the corresponding quantities $I_{0, \mathrm{TE} / \mathrm{TM}}$ in the absence of the cylinders $(a=0)$. The exact shape of the curves Fig. 3 is of course dependent on the random characteristics of the sources, and that is why rapid "noisy" oscillations are observed; however, the waveform trend converges for a large number of dipoles [points of integration (7) along the interval $0<Z<d$ ]. Again, two operational wavelengths are considered for the blue light [Fig. 3(a)] and red light [Fig. 3(b)], respectively, while the size $b$ is the same as in Fig. 2 . Note that the normalization intensity $I_{0}$ is only dependent on the wavelength $\lambda_{0}$ and the type of polarization; therefore, it seems fitting (since it is constant) for the optimization with respect to various dimensions $(a, b)$. We also show the average $I_{T E+\mathrm{TM}}=\left(I_{\mathrm{TE}}+\right.$ $\left.I_{\mathrm{TM}}\right) / 2$ of the two normalized curves, describing a case of QWs in which the polarization of each dipole is uncertain with equal probability (50\%) of being TE or TM. Obviously, all the curves take unitary values at $a=0$. In Fig. 3(a), where $\lambda_{0}=450 \mathrm{~nm}$, the normalized quantities fluctuate around unity for small $a / b$ and drop rapidly from $a \cong 0.7 b$. Accordingly, its magnitude vanishes for a range of $a / b$ around $a \cong 0.8 b$ (center of blue blockage zone) before increasing again for larger $a / b$. This negligible-response region corresponds to the bandgap of the effective media shown in Fig. 2(a), which prevents the propagation of the waves produced by the incoherent dipoles of the region $0<z<d$.

In Fig. 3(b), we show the radiated intensity with respect to $a / b$ for red light $\left(\lambda_{0}=630 \mathrm{~nm}\right)$, where the decreasing parabolic trend is imposed mainly by the factor $(1-f)=$ $\left(1-\frac{\pi}{4}(a / b)^{2}\right)$, as shown in Eqs. (7) and (8). In other words, the contribution of the integral over $Z$ is not that significant to the total output compared to the volume factor $(1-f)$ due to the dielectric character of the equivalent material [with response determined by the parameters of Fig. 2(b)]. A secondary reason for the reduced [but nonzero, as in Fig. 3(a)] light emission is the total internal reflection, which becomes stronger since the effective permittivity contrast at the interface with vacuum increases according to Fig. 2(b). On the contrary, the forbidden zone indicated by the plasmonic character of

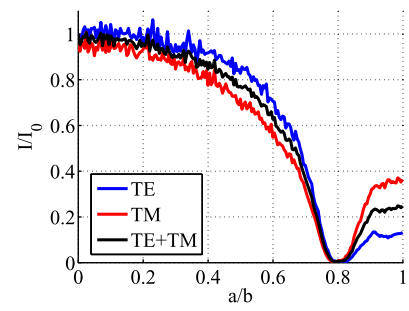

(a)

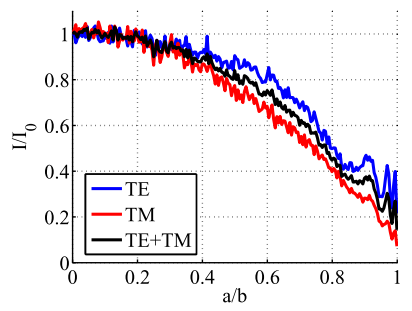

(b)
Fig. 3. Normalized radiation intensities $I_{\mathrm{TE} / \mathrm{TM}}^{\mathrm{blue} / \mathrm{red}} / I_{0, \mathrm{TE} / \mathrm{TM}}^{\mathrm{blue} / \mathrm{red}}$ as functions of $a / b$ for (a) blue light $\left(\lambda_{0}=450 \mathrm{~nm}\right)$ and (b) red light $\left(\lambda_{0}=630 \mathrm{~nm}\right)$. Plot parameters: $b=110 \mathrm{~nm}$. 
the effective medium in Fig. 2(a) is translated into negligible intensity for a certain range of $a / b$ in Fig. 3(a) despite the fact that $(1-f)$ is non-vanishing.

\section{B. Strategy and Optimization}

Our strategy in order to give optimal LED designs [31] is to select the dimensions $a$ and $b$ in order to suppress the radiation extraction for the blue light and enhance the device response for the red light. In this way, the pumped energy at $\lambda_{0}=450 \mathrm{~nm}$ by the incoherent dipoles would have no alternative but to concentrate into the cylinders with QDs, where the color conversion occurs, and get radiated from the structure in the form of red light. If a design contains regions where hole-electron recombination (blue-red color converter) is supported and blocks the blue color while favoring red-color radiation, then naturally, when it is fed by blue-colored waves, it will maximally produce red-colored ones [17]. Therefore, the metrics that should be maximized are the normalized intensities for redlight excitation over the corresponding quantity for blue light:

$$
M_{\mathrm{TE} / \mathrm{TM}}=\frac{I_{\mathrm{TE} / \mathrm{TM}}^{\text {red }} / I_{0, \mathrm{TE} / \mathrm{TM}}^{\text {red }}}{I_{\mathrm{TE} / \mathrm{TM}}^{\text {blue }} / I_{0, \mathrm{TE} / \mathrm{TM}}^{\text {blue }}} .
$$

The average ratio $M=\left(M_{\mathrm{TE}}+M_{\mathrm{TM}}\right) / 2$ for the case of Fig. 3 is maximized for $a / b \cong 0.79$, which corresponds to the center of the blockage band in Fig. 3(a).

In Fig. 4, we show the polar plots (across the upper half space $0<\varphi<\pi)$ and normalized radiated powers $P_{\mathrm{TE} / \mathrm{TM}}(\varphi) /$ $I_{0, \mathrm{TE} / \mathrm{TM}}$ (multiplied by the same large radius $r$ as in Eq. (6) to neutralize its effect) for both polarizations and regarded frequencies $\left[\lambda_{0}=450 \mathrm{~nm}\right.$ for Figs. $4(\mathrm{a})$ and $4(\mathrm{c})$ and $\lambda_{0}=$ $630 \mathrm{~nm}$ for Figs. 4(b) and 4(d)]. Two configurations are considered: the QDs-free structure $[a=0$ in Figs. 4(a) and 4(b)] and the one with optimal $a / b[a / b \cong 0.79$ in Figs. $4(\mathrm{c})$ and $4(\mathrm{~d})$ ]. In the absence of the cylinders, the relative radiation for blue light [Fig. 4(a)] is similar for both polarizations, and numerous sidelobes are recorded away from the maximal direction $\varphi=\pi / 2$. The same happens under the excitation of red light [Fig. 4(b)], but less rapid azimuthal variations are observed. In other words, the structure without the photonic crystals treats similarly the blue and the red-colored excitation, despite

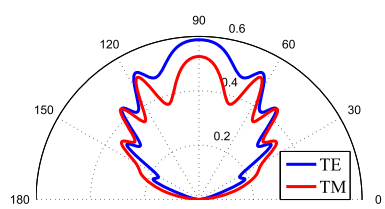

(a)

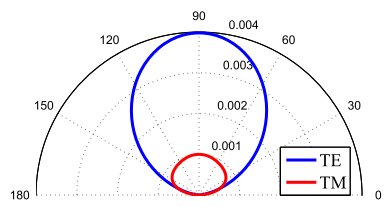

(c)

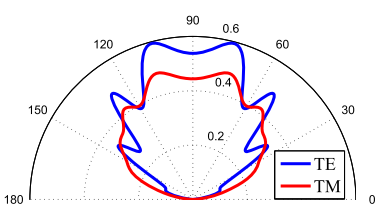

(b)

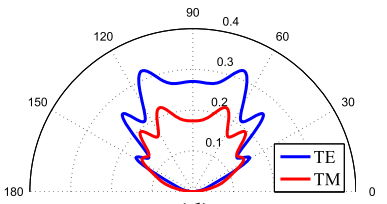

(d)
Fig. 4. Normalized radiated powers $P_{\mathrm{TE} / \mathrm{TM}}^{\mathrm{blue}}(\varphi) / I_{0 \text {, TE/TM }}^{\mathrm{blue} / \mathrm{red}}$ multiplied by $r$, in contour plots across $0<\varphi<\pi$ for (a) blue light, $a=0$; (b) red light, $a=0$; (c) blue light, optimal $a \cong 0.79 b$; and (d) red light, optimal $a \cong 0.79 b$. Plot parameters: $b=110 \mathrm{~nm}$. potential differences in azimuthal profiles originating from the random complex amplitudes $J(Z)$ and $V(Z)$ of the numerical integrations (7) and (8). On the contrary, the radiation patterns of Fig. 4(c), which are normalized by the same quantities $I_{0, \mathrm{TE} / \mathrm{TM}}$ as in Fig. 4(a), are of negligible magnitude, which means that the device for the optimal $a / b$ traps blue light and does not let it leak into free space. The same configuration favors red light since the responses of Fig. 4(d) are comparable to those of Fig. 4(b) with the same normalization constants $I_{0, \mathrm{TE} / \mathrm{TM}}$. Therefore, if the multiple QWs pump that structure (with $a / b \cong 0.79$ ) with energy concentrated at the blue part of visible spectrum, the fields will find the way out by exploiting the color-converting mechanism operating into the cylinders. In particular, they will increase the power concentration into the photonic crystals, enhance the hole-electron recombination, and get radiated in the form of red color.

Up to this point, we have assumed that the half-size of the cell is kept fixed at $b=110 \mathrm{~nm}$. However, the lattice period $2 b$ is an important parameter which should be optimized simultaneously with the radius $a$ of the cylinders. In order to do so, the quantities $I_{\mathrm{TE} / \mathrm{TM}}^{\mathrm{blu} / \text { red }}, M_{\mathrm{TE} / \mathrm{TM}}$ should be represented on a map where the ratio of the distances $a / b$ corresponds to the horizontal axis and the PhCs' spatial period $b$ corresponds to the vertical one. That would be the basic tool for the optimization of the defined structure. Different designs with constant radii of cylinders $a$ are shown as isosceles hyperbolas on the plane $(a / b, b)$ since $b=\frac{a}{a / b}$. Obviously, $0<a / b<1$, while $b$ is kept smaller than $160 \mathrm{~nm}$ to assist homogenization.

In Fig. 5, we show the contour plots of the normalized intensities $I_{\mathrm{TE} / \mathrm{TM}} / I_{0, \mathrm{TE} / \mathrm{TM}}$ for the two operational wavelengths $\lambda_{0}=450,630 \mathrm{~nm}$ and both polarizations. As far as the blue-light excitation is concerned [Figs. 5(a) and 5(b)], we notice that if $a / b$ is low, the result is not significantly affected by the selection of half-size $b$ of the cell. This is a natural result since, for thin $\mathrm{PhCs}$, the response is close to that of unloaded QW multilayers characterized by the equivalent permittivities of Eq. (1) regardless of the dipole polarization. For

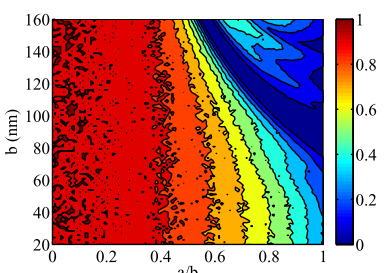

(a)

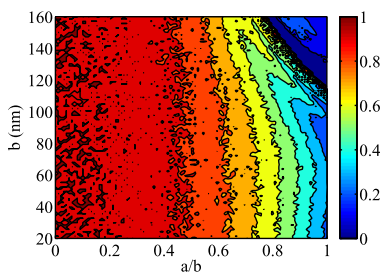

(c)

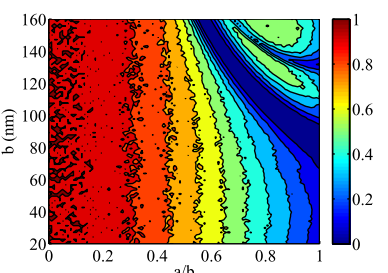

(b)

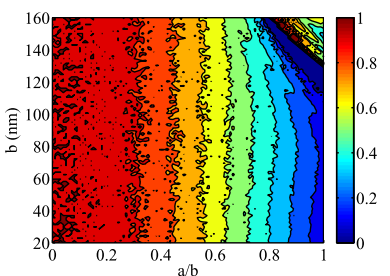

(d)
Fig. 5. Normalized intensities $I_{\mathrm{TE} / \mathrm{TM}}^{\text {blue/red }} / I_{0, \mathrm{TE} / \mathrm{TM}}^{\text {blue/red }}$ represented on $(a / b, b)$ plane for (a) TE polarization, blue light; (b) TM polarization, blue light; (c) TE polarization, red light; and (d) TM polarization, red light. 
$a / b$ higher than a threshold of $a / b \cong 0.6$, a bandgap leading to vanishing radiation extraction appears for a range of $b$ values which correspond to plasmonic behavior of the effective medium of Eqs. (2) and (3). This $b$-interval becomes more sizable and concerns smaller average values as $a / b$ gets larger. On the contrary, the intensity for the red light [Figs. 5(c) and 5 (d)] decreases more reluctantly with respect to $a / b$ and is certainly non-vanishing across the aforementioned plasmonic zones of Figs. 5(a) and 5(b). In this way, the functional combinations of $a / b$ and $b$ are those leading to an effective medium (for the blue color) with either negative transversal permittivity $\left(\operatorname{Re}\left[\varepsilon_{x}=\varepsilon_{y}\right]\right)$ or permeability $\left(\operatorname{Re}\left[\mu_{x}=\mu_{y}\right]\right)$ which are the most significant ones. Indeed, due to the way we excite the structure, waves traveling mainly towards the $z$ axis are developed; furthermore, the finite size of the layers along the $z$ directions restricts the role of longitudinal effective parameters $\left(\varepsilon_{z}, \mu_{z}\right)$.

In Fig. 6, we represent the metrics $10 \log \left(1+M_{\mathrm{TE} / \mathrm{TM}}\right)$ (in $\mathrm{dB})$ for both polarizations, demonstrating how more substantial the normalized radiation extraction is for the red light compared to the one of blue light on the $(a / b, b)$ plane. It is clear that the large values either for the TE or TM cases are concentrated across a strip at the upper right part of our map. We can approximately find that the highest performance concerns the following range for the radius of the cylinders: $80 \mathrm{~nm}<a<120 \mathrm{~nm}$ (with $a<b<160 \mathrm{~nm}$, as we have assumed). Maps of the metrics $M_{\mathrm{TE}}$ and $M_{\mathrm{TM}}$ like the ones depicted in Fig. 6, or variations of the average of them $M=\left(M_{\mathrm{TE}}+M_{\mathrm{TM}}\right) / 2$ as long as the events of TE or TM radiation happen with the same probability, can be extremely helpful for the designer of similar LED systems. Note that the shape of this maximal $M_{\mathrm{TE} / \mathrm{TM}}$ zone does not correspond to a fixed radius $a$ despite the fact that it has a hyperbolic shape. However, one observes that the color conversion is maximized within almost the same regions of the plane $(a / b, b)$ regardless of the polarization and thus numerous alternative optimal configurations can be obtained. Such a finding can restrict substantially the parametric space of the considered LED coloring designs and simplify significantly the subsequent optimizations. Most importantly, if one is able to find, without computationally heavy schemes $[31,41]$, a set with several candidate designs exhibiting ultra-high performance, one can perform a secondary sweep within this set by posing extra constraints or demands (cost, ease of fabrication, durability, etc.).

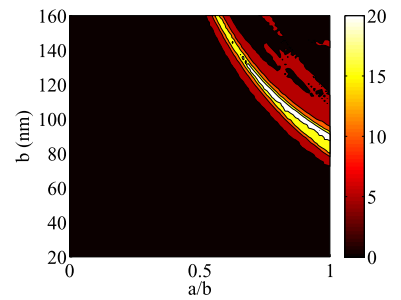

(a)

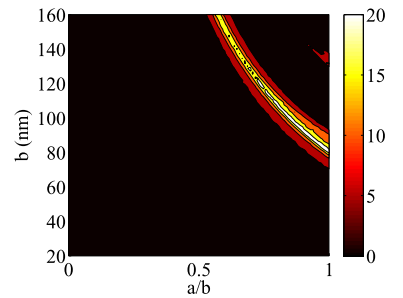

(b)
Fig. 6. Metrics $10 \log \left(1+M_{\mathrm{TE} / \mathrm{TM}}\right)$ expressed in $\mathrm{dB}$ and represented on $(a / b, b)$ plane for (a) TE polarization and (b) TM polarization.

\section{CONCLUSIONS}

Optimization of LED structures incorporating photonic crystals for maximum light extraction is a computationally challenging task since the diode is fed by randomly oriented and excited incoherent sources. In this work, we employed effective medium theory for the regions containing the sources and the cylinders; thus, we rendered the formulated boundary value problem analytically solvable. In this way, we obtained convenient approximate formulas for the far-field radiation of the structure and defined suitable metrics characterizing the color-conversion performance of the device. By optimizing these metrics, we proposed numerous alternative designs which favor the transformation of blue to red light and can serve as initial points to find ultraefficient combinations of periodic lattices and planar background geometries. In the vicinity of these initial points, one can search for maximum actual performances for the considered structures based on accurate, full-wave simulations provided by commercial packages.

The analyzed method can be easily applied to different color conversions and generalized to take into account arbitrary numbers of layers or inhomogeneities contained in the structural configuration. In addition, it can be further refined with help from more sophisticated effective medium approximations, giving full permittivity and permeability tensors for the equivalent homogeneous medium. An interesting expansion of the present work would be to consider additional constraints such as the spatial distribution of the field at the cross section of the photonic crystals, the microscopic characteristics of the quantum dot particles, or the interaction with neighboring components, leading to competitive candidate designs for LED-based integrated systems.

Funding. Newton Fund (216425309); Ministry of Education and Science of the Republic of Kazakhstan (BR05236454); Nazarbayev University Small Grants (17095).

Acknowledgment. This work was partially supported by the Newton-Al-Farabi Partnership Program under the project entitled "Designing energy-efficient and highly controllable photonic structures," and by Nazarbayev University Small Grants under the project entitled "Super transmitters, radiators and lenses via photonic synthetic matter". Funding from the MES RK state-targeted program is also acknowledged.

\section{REFERENCES}

1. E. F. Schubert, Light-Emitting Diodes (Cambridge University, 2014).

2. G. Stringfellow and M. Craford, High Brightness Light Emitting Diodes, Semiconductor and Semimetals (Academic, 1997), Vol. 48.

3. G. Held, Introduction to Light Emitting Diode Technology and Applications (CRC Press/Taylor \& Francis, 2016).

4. S. Reineke, M. Thomschke, B. Lussem, and K. Leo, "White organic light-emitting diodes: status and perspective," Rev. Mod. Phys. 85, 1245-1293 (2013).

5. Y. Shirasaki, G. J. Supran, M. G. Bawendi, and V. Bulovic, "Emergence of colloidal quantum-dot light-emitting technologies," Nat. Photonics 7, 13-23 (2013).

6. B. S. Mashford, M. Stevenson, Z. Popovic, C. Hamilton, Z. Zhou, C. Breen, J. Steckel, V. Bulovic, M. Bawendi, S. C. Coe-Sullivan, and P. T. Kazlas, "High-efficiency quantum-dot light-emitting devices with enhanced charge injection," Nat. Photonics 7, 407-412 (2013). 
7. X. Dai, Z. Zhang, Y. Jin, Y. Niu, H. Cao, X. Liang, L. Chen, J. Wang, and $X$. Peng, "Solution-processed, high-performance light-emitting diodes based on quantum dots," Nature 515, 96-99 (2014).

8. S. A. Veldhuis, P. P. Boix, N. Yantara, M. Li, T. C. Sum, N. Mathews, and S. G. Mhaisalkar, "Perovskite materials for light-emitting diodes and lasers," Adv. Mater. 28, 6804-6834 (2016).

9. Z. Yin, X. Liu, Y. Wu, X. Hao, and X. Xu, "Enhancement of light extraction in GaN-based light-emitting diodes using rough beveled ZnO nanocone arrays," Opt. Express 20, 1013-1021 (2012).

10. Y. Yang, Y. Zheng, W. Cao, A. Titov, J. Hyvonen, J. R. Manders, J. Xue, P. H. Holloway, and L. Qian, "High-efficiency light-emitting devices based on quantum dots with tailored nanostructures," Nat. Photonics 9, 259-266 (2015).

11. M. V. Kovalenko, L. Manna, A. Cabot, Z. Hens, D. V. Talapin, C. R. Kagan, V. I. Klimov, A. L. Rogach, P. Reiss, D. J. Milliron, P. GuyotSionnnest, G. Konstantatos, W. J. Parak, T. Hyeon, B. A. Korgel, C. B. Murray, and W. Heiss, "Prospects of nanoscience with nanocrystals," ACS Nano 9, 1012-1057 (2015).

12. F. Priolo, T. Gregorkiewicz, M. Galli, and T. F. Krauss, "Silicon nanostructures for photonics and photovoltaics," Nat. Nanotechnol. 2, 515-520 (2007).

13. M. E. Zoorob, M. D. B. Charlton, G. J. Parker, J. J. Baumberg, and M. C. Netti, "Complete photonic bandgaps in 12-fold symmetric quasicrystals," Nature 404, 740-743 (2000).

14. F. S. Diana, A. David, I. Meinel, R. Sharma, C. Weisbuch, S. Nakamura, and P. M. Petroff, "Photonic crystal-assisted light extraction from a colloidal quantum dot/GaN hybrid structure," Nano Lett. 6, 1116-1120 (2006).

15. Y. C. Shin, D. H. Kim, E. H. Kim, J. M. Park, K. M. Ho, K. Constant, J. H. Choe, Q. H. Park, H. Y. Ryu, J. H. Baek, T. Jung, and T. G. Kim, "High efficiency GaN light-emitting diodes with two dimensional photonic crystal structures of deep-hole square lattices," IEEE J. Quantum Electron. 46, 116-120 (2010).

16. N. Ganesh, W. Zhang, P. C. Mathias, E. Chow, J. A. N. T. Soares, V. Malyarchuk, A. D. Smith, and B. T. Cunningham, "Enhanced fluorescence emission from quantum dots on a photonic crystal surface," Nat. Nanotechnol. 2, 515-520 (2007).

17. J. J. Wierer, A. David, and M. M. Megens, "III-nitride photonic-crystal light-emitting diodes with high extraction efficiency," Nat. Photonics 3 , 163-169 (2009).

18. S. Chanyawadee, P. G. Lagoudakis, R. T. Harley, M. D. B. Charlton, D. V. Talapin, H. W. Huang, and C. H. Lin, "Increased colorconversion efficiency in hybrid light-emitting diodes utilizing nonradiative energy transfer," Adv. Mater. 22, 602-606 (2010).

19. A. David, T. Fujii, R. Sharma, K. McGroddy, S. Nakamura, S. P. Den Baars, E. L. Hu, C. Weisbuch, and H. Benisty, "Photonic-crystal GaN light-emitting diodes with tailored guided modes distribution," Appl. Phys. Lett. 88, 061124 (2006).

20. C. Wiesmann, K. Bergenek, N. Linder, and U. T. Schwarz, "Photonic crystal LEDs-designing light extraction," Laser Photon. Rev. 3, 262-286 (2009).

21. F. Zhang, J. Liu, G. You, C. Zhang, S. E. Mohney, M. J. Park, J. S. Kwak, Y. Wang, D. D. Koleske, and J. Xu, "Nonradiative energy transfer between colloidal quantum dot-phosphors and nanopillar nitride LEDs," Opt. Express 20, A333-A339 (2012).
22. C. Krishnan, M. Brossard, K.-Y. Lee, J.-K. Huang, C.-H. Lin, H.-C. Kuo, M. D. B. Charlton, and P. G. Lagoudakis, "Hybrid photonic crystal light-emitting diode renders $123 \%$ color conversion effective quantum yield," Optica 3, 503-509 (2016).

23. E. Kallos, I. Chremmos, and V. Yannopapas, "Resonance properties of optical all-dielectric metamaterials using two-dimensional multipole expansion," Phys. Rev. B 86, 245108 (2012).

24. C. A. Valagiannopoulos, "Closed-form solution to the scattering of a skew strip field by metallic pin in a slab," Prog. Electromagn. Res. 79, 1-21 (2008).

25. O. T. A. Janssen, A. J. H. Wachters, and H. P. Urbach, "Efficient optimization method for the light extraction from periodically modulated LEDs using reciprocity," Opt. Express 18, 24522-24535 (2010).

26. A. H. Sihvola, Electromagnetic Mixing Formulas and Applications, IEEE Electromagnetic Waves Series (IET, 1999), Vol. 47.

27. S. Zouhdi, A. Sihvola, and A. P. Vinogradov, Metamaterials and Plasmonics: Fundamentals, Modelling, Applications (Springer, 2009).

28. A. Alu, "First-principles homogenization theory for periodic metamaterials," Phys. Rev. B 84, 075153 (2011).

29. X. Hu, C. T. Chan, J. Zi, M. Li, and K. M. Ho, "Diamagnetic response of metallic photonic crystals at infrared and visible frequencies," Phys. Rev. Lett. 96, 223901 (2006).

30. A. Campa, J. Krc, and M. Topic, "Two approaches for incoherent propagation of light in rigorous numerical simulations," Prog. Electromagn. Res. 137, 187-202 (2013).

31. M. D. B. Charlton, M. E. Zoorob, and T. Lee, "Photonic quasi-crystal LEDs: design, modelling, and optimisation," Proc. SPIE 6486, 64860R (2007).

32. I. Chremmos, E. Kallos, M. Giamalaki, V. Yannopapas, and E. Paspalakis, "Effective medium theory for two-dimensional nonmagnetic metamaterial lattices up to quadrupole expansions," J. Opt. 17, 075102 (2015).

33. C. A. Valagiannopoulos, "Study of an electrically anisotropic cylinder excited magnetically by a straight strip line," Prog. Electromagn. Res. 73, 297-325 (2007).

34. J. Shakya, K. Knabe, K. H. Kim, J. Li, J. Y. Lin, and H. X. Jianga, "Polarization of III-nitride blue and ultraviolet light-emitting diodes," Appl. Phys. Lett. 86, 091107 (2005).

35. A. Erdelyi, Asymptotic Expansions (Dover, 1956)

36. C. A. Balanis, Advanced Engineering Electromagnetics (Dover, 1972).

37. D. Marcuse, "Excitation of parabolic-index fibers with incoherent sources," Bell Syst. Tech. J. 54, 1507-1530 (1975).

38. M. N. Polyanskiy, "Refractive index database," http://refractiveindex info.

39. M. M. Y. Leung, A. B. Djurisic, and E. H. Li, "Refractive index of InGaN/ GaN quantum well," J. Appl. Phys. 84, 6312-6317 (1998).

40. J. B. Pendry, L. Martın-Moreno, and F. J. Garcia-Vidal, "Mimicking surface plasmons with structured surfaces," Science 305, 847-848 (2004).

41. J. Michallon, D. Bucci, A. Morand, M. Zanuccoli, V. Consonni, and A. Kaminski-Cachopo, "Light absorption processes and optimization of $\mathrm{ZnO} / \mathrm{CdTe}$ core-shell nanowire arrays for nanostructured solar cells," Nanotechnology 26, 075401 (2015). 